\title{
Feasibility and safety of third-space endoscopic full-thickness resection in ex vivo and in vivo porcine models $\square$
}

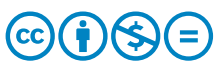

\author{
Authors \\ Naohisa Yahagi² \\ Institutions \\ 1 Department of Gastroenterology, Nippon Medical \\ School, Tokyo, Japan \\ 2 Division of Research and Development for Minimally \\ Invasive Treatment, Cancer Center, Keio University, \\ School of Medicine, Tokyo, Japan
}

Osamu Goto ${ }^{1,2}$, Motoki Sasaki ${ }^{2}$, Teppei Akimoto ${ }^{1,2}$, Atsushi Tatsuguchi ${ }^{1}$, Mitsuru Kaise ${ }^{1}$, Katsuhiko Iwakiri ${ }^{1}$,

submitted 10.8.2018

accepted after revision $\quad 29.10 .2018$

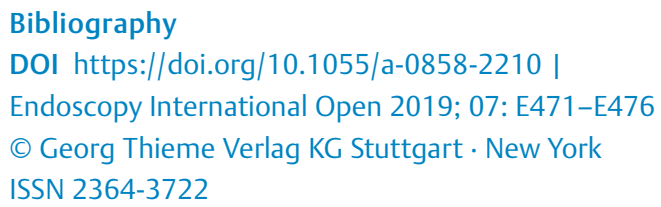

Corresponding author

Osamu Goto, MD, PhD, Department of Gastroenterology, Nippon Medical School, 1-1-5, Sendagi, Bunkyo-ku,

Tokyo 113-8603, Japan

Fax: +81-3-5814-6289

o-goto@nms.ac.jp

\section{ABSTRACT}

Background and study aims Endoscopic full-thickness resection (EFTR) involves several technical issues that need to be addressed. We devised a novel technique termed third-space EFTR and investigated its feasibility and safety in animal models.

Methods Third-space EFTR was performed in three isolated porcine stomachs (ex vivo) and four live pigs (in vivo, 1week survival). The technique involved a circumferential mucosal incision, submucosal tunnelling on the proximal side, endoscopic suturing of the surrounding mucosa, a circumferential seromuscular incision in the submucosal tunnel, transoral retrieval and entry site closure of the tunnel. The technical outcomes were investigated.

Results In the ex vivo study, the procedure was successfully completed with $\mathrm{R} 0$ resection. In the in vivo study, the procedure was completed in all pigs; however, R0 resection failed in one pig owing to snaring resection. All pigs survived without severe adverse events.

Conclusions Our findings indicate that third-space EFTR is feasible and safe. This technique may be useful as a minimally invasive endoscopic option for reliable treatment of small gastric submucosal tumours.

\section{Introduction}

Endoscopic full-thickness resection (EFTR) is considered one of the less invasive endoluminal surgeries for gastric neoplasms such as gastrointestinal stromal tumours (GISTs) [1-3]. However, it has several technical issues that are difficult to address. For example, conventional EFTR involves intentional perforation to resect a lesion in a full-layered manner, which can cause air leakage and deflation of the stomach, and management of the endoscope in a collapsed stomach is difficult owing to poor endoscopic visualisation. Furthermore, secure endoscopic closure of a wall defect after resection is technically demanding, particularly in a deflated stomach. Various closure methods have been proposed so far [4-6]; however, most of these methods are still in development, and hence, have been infrequently introduced in a clinical setting.
Endoscopic resection with submucosal tunnelling has been indicated for intraluminally growing submucosal tumours in the esophagus or stomach [7-9]. However, this technique innately involves enucleation surgery without adequate safety margins; therefore, unexpected injury of the capsule around the tumor can occur, which might cause tumour seeding through the seromuscular defect.

To avoid these drawbacks in conventional EFTR and the endoscopic enucleation technique, we developed a novel EFTR approach termed third-space EFTR. With this novel approach, lesions can be removed transorally in a full-thickness manner without collapse of the stomach or a decrease in endoscopic visualization. The current study aimed to demonstrate the feasibility and safety of third-space EFTR in ex vivo and in vivo porcine survival models. 


\section{Methods}

\section{Settings}

The ex vivo study included three porcine stomachs isolated from pigs used for food. After rinsing the insides of the stomachs with tap water, they were set in a training kit for endoscopic submucosal dissection (ESD). In addition, endoscopic instruments were prepared for ESD and endoscopic hand suturing (EHS), as described elsewhere $[10,11]$. A simulating lesion approximately $3 \mathrm{~cm}$ in diameter was created in each stomach by simply placing mucosal markings.

The in vivo porcine survival study was performed after obtaining approval from the institutional review board of our animal laboratory. The study included four live pigs weighing approximately $20 \mathrm{~kg}$. Under general anaesthesia, third-space EFTR was performed as mentioned below. As in the ex vivo study, a gastric lesion was created in each of the pigs. After completion of the procedure, on waking up, the pigs were allowed to drink water and eat soft food on postoperative day (POD) 1 , followed by normal food on POD 2. We administered $0.5 \mathrm{~g}$ of cefazolin sodium hydrate which was intramuscularly injected just before the procedure and $0.5 \mathrm{~g}$ of cefalexin with diet from postoperative Day 1 until Day 3. Proton pump inhibitor was not administered perioperatively. The pigs were carefully monitored thereafter. On POD 7, they were endoscopically observed and killed for autopsy.

\section{Third-space EFTR}

Third-space EFTR involved several endoscopic procedures ( $\triangleright$ Fig.1). First, a circumferential mucosal incision was made around the lesion using the DualKnife (KD-650-L; Olympus Co. Ltd, Tokyo, Japan) after submucosal injection, and then the submucosa on the lateral side was undermined to enlarge the submucosal space. Second, a submucosal tunnel 3 to $4 \mathrm{~cm}$ in length was created on the proximal side of the lesion by removing the proximal mucosa with the ESD technique and suturing the lateral mucosa with the EHS technique in the shape of a tunnel or by simply using the per-oral endoscopic myotomy (POEM) technique [12]. Third, the mucosa surrounding the lesion was linearly sutured using the EHS technique, with the lesion extruded towards the outside. Fourth, submucosal dissection was performed around the lesion through the submucosal tunnel to broaden the "third space" around the lesion until the endoscope could smoothly approach the muscular layer around the lesion. Fifth, a seromuscular incision was made circumferentially to remove the lesion, and it was transorally retrieved through the submucosal tunnel. The procedure was completed with entry site closure using the EHS technique or clipping.

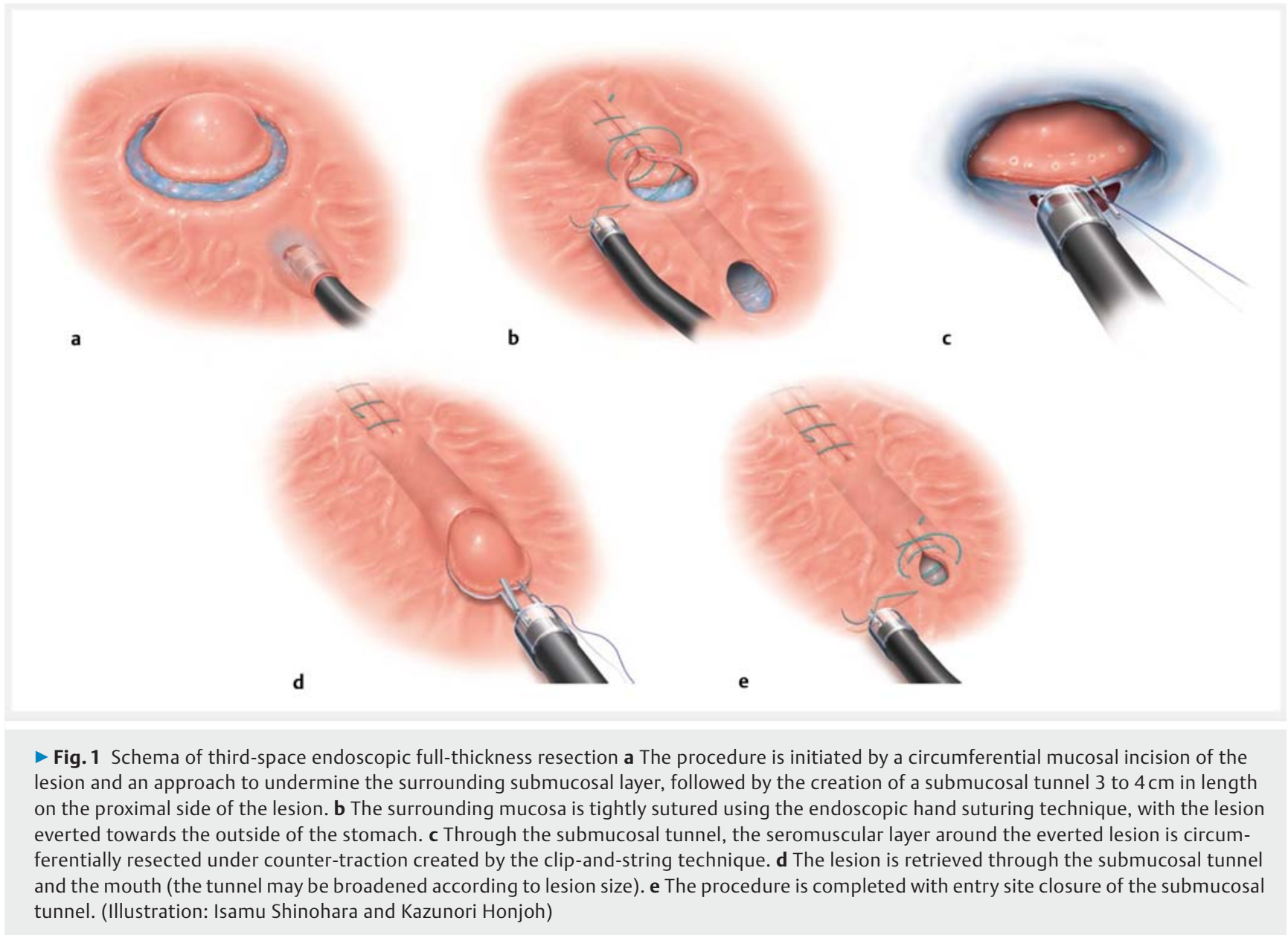




\section{Outcome measures}

As technical outcomes, completeness of the procedure, achievement of R0 resection and procedure duration were investigated in both the ex vivo and in vivo studies. Furthermore, the amount of intraoperative bleeding, occurrence of intraoperative and postoperative adverse events (AEs), 1-week survival, existence of anastomotic leakage at POD 7, post mortem gross findings in the abdominal cavity, and histological findings at the surgical site were assessed in the in vivo study.

\section{Results}

\section{Ex vivo study}

A submucosal tunnel was created by suturing both edges of the mucosal defect at the proximal side. The procedure was successfully completed with $\mathrm{R} 0$ resection using only the DualKnife $(\triangleright$ Table 1). Mean duration of the procedure was $124.3 \pm 11.6$ minutes. In addition, mean maximum sizes of the mucosal and serosal sides were $32 \mathrm{~mm}$ and $34 \mathrm{~mm}$, respectively.

\section{In vivo survival study}

This study included four pigs. In the first two pigs, a submucosal tunnel was created in the same manner as in the ex vivo study. However, the suture site on the roof of the tunnel partially tore because of pressure from the endoscope at the seromuscular incision in the first pig. The damage was intraoperatively repaired with repeated EHS, and there were no issues. Therefore, the tunnel was created using the POEM technique in the latter two pigs. The procedure was completed in all pigs. Snaring resection was conducted in the first three cases. However, R0 resection failed in the third pig owing to blind resection by snaring because the lesion moved to the distal side from the endoscope as we proceeded with the incision due to lack of counter-traction and the accurate incision line became difficult to recognize. Therefore, in the fourth pig, counter-traction was utilized by pulling the proximal edge of the lesion using the clip-with-string technique [13], which led to optimal and successful en-bloc resection using only the DualKnife (
- Video 1). Mean duration of the procedure was $136.3 \pm 29.4$ minutes ( $\triangleright$ Table $\mathbf{1}$ ). In addition, mean maximum sizes of the mucosal and serosal sides were $31 \mathrm{~mm}$ and $23 \mathrm{~mm}$, respectively. In all cases, no severe bleeding occurred, and minor bleeding events were stopped spontaneously or by coagulation with the tip of the electrocautery knife.

Pneumoperitoneum was inevitable in all cases; however, vital signs were stable during the procedure, therefore, no abdominal puncture was required. All the pigs survived without severe AEs. Endoscopy on POD 7 showed no anastomotic leakage in all the pigs ( $>$ Fig. 3a). A small amount of ascites with a localized abscess at the wall defect was observed. There was no fistula owing to firm attachment of the greater omentum ( Fig.3b, $>$ Fig.3c). Histologically, granulomatous tissue bridged the wall defect with attachment of the omentum ( $\triangleright$ Fig.3d). There was no apparent transmural communication. These changes were found in all pigs and no apparent differences were observed.

\section{Discussion}

In our two consecutive animal studies, we successfully demonstrated that third-space EFTR was feasible and safe. With this technique, which does not involve direct perforation and therefore does not involve deflation of the lumen, secure and optimal full-layered resection is expected endoscopically, without a decrease in endoscopic visualization and a subsequent decrease in endoscopic maneuverability. Accordingly, this technique may be a secure and minimally invasive endoscopic option for transorally retrievable submucosal tumors.

The three main components of third-space EFTR are mucosal incision and suturing, tunnelling and seromuscular incision. Initiation of mucosal suturing before the seromuscular incision is important to avoid full-layer perforation. Submucosal tunnelling is necessary to approach the lesion for the seromuscular incision after mucosal suturing and to avoid deflation of the lumen [14]. When compared with conventional EFTR, third-space EFTR additionally requires submucosal tunnelling, and it is mandatory to overcome the drawbacks of conventional EFTR.

- Table 1 Ex vivo study details.

\begin{tabular}{|c|c|c|c|c|c|c|c|c|c|}
\hline Cases & $\begin{array}{l}\text { Loca- } \\
\text { tion }\end{array}$ & $\begin{array}{l}\text { Circum- } \\
\text { ference }\end{array}$ & $\begin{array}{l}\text { Procedure } \\
\text { completeness }\end{array}$ & $\begin{array}{l}\text { RO re- } \\
\text { section }\end{array}$ & $\begin{array}{l}\text { Total proce- } \\
\text { dural time } \\
\text { (min) }\end{array}$ & $\begin{array}{l}\text { Time for } \\
\text { hand sutur- } \\
\text { ing (min) }\end{array}$ & $\begin{array}{l}\text { Mucosal } \\
\text { size } \\
(\mathrm{mm} \times \mathbf{m m})\end{array}$ & $\begin{array}{l}\text { Serosal } \\
\text { size }(\mathrm{mm} \times \\
\mathrm{mm})\end{array}$ & $\begin{array}{l}\text { 1-week } \\
\text { survival }\end{array}$ \\
\hline Ex vivo 1 & M & Post & Completed & Yes & 112 & 48 & $30 \times 20$ & $40 \times 20$ & \\
\hline Ex vivo 2 & M & Less & Completed & Yes & 135 & 48 & $40 \times 25$ & $38 \times 22$ & \\
\hline Ex vivo 3 & M & Ant & Completed & Yes & 126 & 53 & $25 \times 24$ & $25 \times 23$ & \\
\hline In vivo 1 & $N$ & Post & Completed & Yes & 157 & 40 & $30 \times 25$ & $25 \times 12$ & Alive \\
\hline In vivo 2 & M & Gre & Completed & Yes & 110 & 38 & $35 \times 32$ & $23 \times 8$ & Alive \\
\hline In vivo 3 & M & Ant & Completed & Yes & 112 & 34 & $20 \times 16$ & $15 \times 9$ & Alive \\
\hline In vivo 4 & $M$ & Post & Completed & Yes & 166 & 24 & $40 \times 40$ & $30 \times 25$ & Alive \\
\hline
\end{tabular}



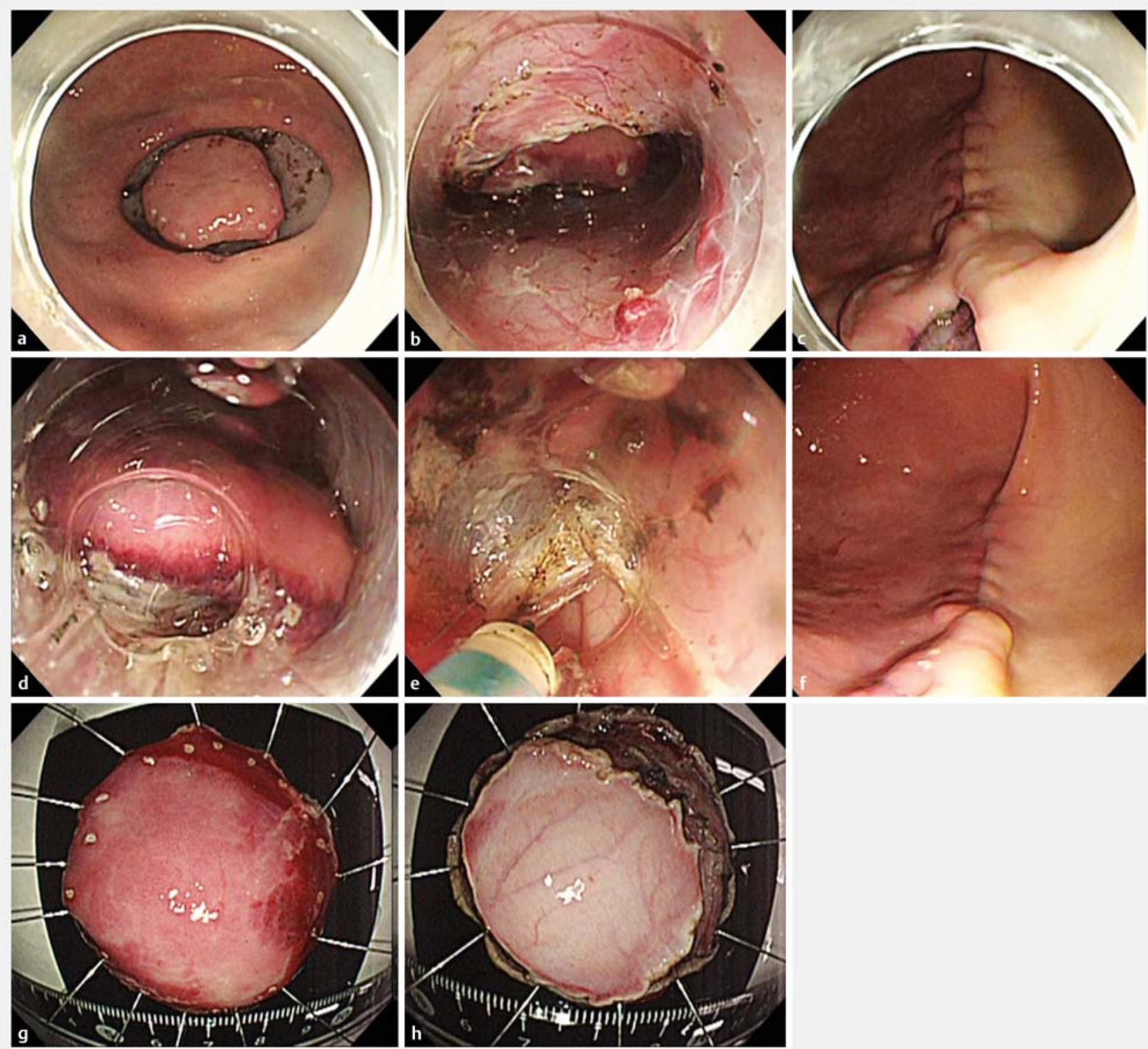

- Fig. 2 Images of third-space endoscopic full-thickness resection in an in vivo porcine model. a A mucosal incision around a simulating lesion is completed circumferentially. Subsequently, submucosal dissection beneath the surrounding mucosa is partially performed to widen the submucosal space. $\mathbf{b}$ A submucosal tunnel 3 to $4 \mathrm{~cm}$ in length is created at the proximal side of the lesion. The oral side of the lesion is seen through the tunnel. $\mathbf{c}$ The mucosal layer around the lesion is closed using the endoscopic hand suturing technique, with the lesion buried. d The buried lesion is visible in the "third space" through the submucosal tunnel. e A seromuscular incision is performed while pulling the lesion with a clip and string. $\mathbf{f}$ After retrieval of the lesion transorally, the entry site of the submucosal tunnel is closed. $\mathbf{g} \mathrm{A}$ mucosal plane of the resected specimen. $\mathbf{h}$ A serosal plane. Full-thickness resection is achieved with a pure endoscopic procedure.

When compared with endoscopic enucleation [7-9], thirdspace EFTR enables removal of the lesion in a full-thickness manner without exposure of the tumor surface. Particularly for a GIST, injury of the capsule around the tumor should be avoided owing to the possibility of tumor cell seeding. In this technique, it is possible to resect the lesion without causing injury to the capsule; however, care should be taken to avoid unintentional rupture of the capsule. Furthermore, submucosal dissection around the entire tumor surface is not required with this technique. Therefore, this technically demanding phase in endoscopic enucleation can be skipped, and the procedural time can be reduced. Obviously, this technique should not be used to treat ulcerated GISTs or early adenocarcinomas because neoplastic cells are already exposed in these lesions and therefore risk of peritoneal seeding may arise by performing this transluminal technique.

In clinical settings, we may have to be careful to prevent damage to the suture site in the seromuscular incision because, unlike the simulating lesions which are flat, SMTs are large and bulging and might lead to tearing of the suture site due to a 


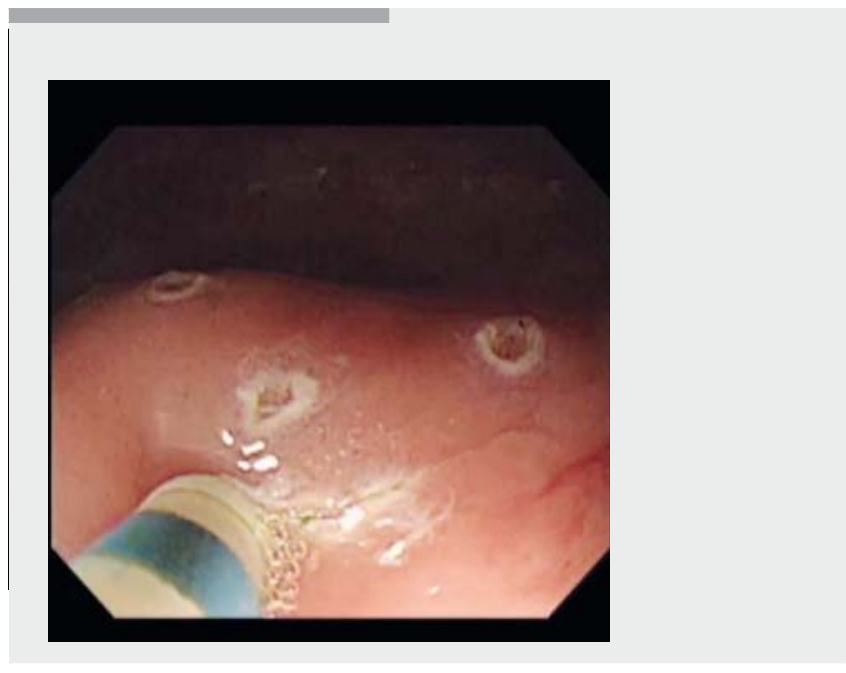

Video 1 Third-space endoscopic full-thickness resection. Third-space endoscopic full-thickness resection involves a circumferential mucosal incision, submucosal tunnelling at the proximal side, a mucosal closure, and a seromuscular incision in the third space. After retrieval of the specimen, the procedure is completed with entry site closure.
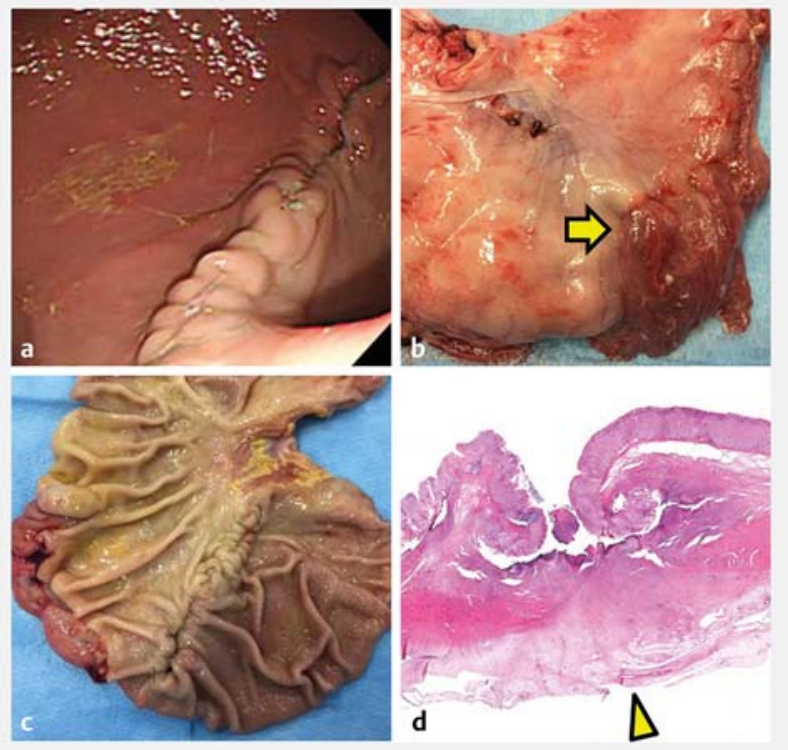

- Fig. 3 Gross and histological findings on postoperative Day 7. a Endoscopic findings. The resection scar and entry site are closed. b Serosal side of the operated site. The seromuscular defect is covered with the greater omentum (arrow). $\mathbf{c}$ Inside of the operated site. No apparent fistula is seen. $\mathbf{d}$ Histology of the anastomotic site. A granulomatous change with massive fibrotic tissue is seen. The greater omentum is patched to the seromuscular defect (arrowhead). narrow working space. Adequate undermining of the submucosal layer and sufficient enlargement of the third-space may be important for a successful procedure in human cases.

Furthermore, ways to treat and avoid massive bleeding in the abdominal space remain a major concern in clinically introducing this procedure. Although we have fortunately not encountered massive bleeding from larger vessels, we may have to be careful not to unintentionally cut larger vessels by taking a short step in the seromuscular incision under clear visualization or by using a scissors-type knife.

Suture-and-cut techniques with the over-the-scope clip can be promising in terms of simplicity and accessibility [15] when the target lesions are small enough. The current technique can target larger lesions, as the resection line can be optimally designed without size limitations of clips or electrocautery snares.

The current study had several limitations. First, it was preclinical with a small number of porcine stomachs. Based on the results in our animal studies, a further clinical study should be performed to investigate the feasibility of third-space EFTR. Second, the current technique leaves a seromuscular defect intact. Although the in vivo study demonstrated 1-week survival of the pigs and previous clinical studies [1-3] have demonstrated the safety of conventional EFTR, which was accompanied by only mucosal closure, the safety of the current technique should be confirmed in clinical settings. Otherwise, seromuscular closure with an available technique [4-6] or a new approach should be considered. Third, it is unclear whether the current technique can be applied to various locations in the stomach, as the technique was performed for optimal lesions in this study. Although we are optimistic about application of the current technique to various locations considering that ESD or other advanced endoscopic surgeries can be performed irrespective of the location, the feasibility of this technique should be confirmed in other locations. Fourth, suturing by the EHS technique has not been available worldwide so far, which makes the technique a little less reproducible.

\section{Conclusion}

In conclusion, third-space EFTR was found to be feasible and safe in porcine models. This reliable EFTR technique can be applied to per-orally retrievable gastric submucosal tumors in a full-thickness manner with adequate surgical margins. A further clinical study is expected in the future.

\section{Acknowledgements}

This study was partly supported by a Japan Consortium for Advanced Surgical Endoscopy (J-CASE) Research Grant. We thank Mr. Isamu Shinohara and Mr. Kazunori Honjoh for creating the illustration of the third-space EFTR procedure.

\section{Competing interests}




\section{References}

[1] Zhou PH, Yao LQ, Qin XY et al. Endoscopic full-thickness resection without laparoscopic assistance for gastric submucosal tumors originated from the muscularis propria. Surg Endosc 2011; 25: 2926 2931

[2] Shi Q, Chen T, Zhong YS et al. Complete closure of large gastric defects after endoscopic full-thickness resection, using endoloop and metallic clip interrupted suture. Endoscopy 2013; 45: 329-334

[3] Ye LP, Zhang Y, Luo DH et al. Safety of endoscopic resection for upper gastrointestinal subepithelial tumors originating from the muscularis propria layer: an analysis of 733 tumors. Am J Gastroenterol 2016; 111: $788-796$

[4] Kantsevoy SV, Bitner M, Mitrakov AA et al. Endoscopic suturing closure of large mucosal defects after endoscopic submucosal dissection is technically feasible, fast, and eliminates the need for hospitalization (with videos). Gastrointest Endosc 2014; 79: 503-507

[5] Schmidt A, Bauder M, Riecken B et al. Endoscopic full-thickness resection of gastric subepithelial tumors: A single-center series. Endoscopy 2015; 47: $154-158$

[6] Mori H, Kobara H, Fujihara S et al. Feasibility of pure EFTR using an innovative new endoscopic suturing device: the double-arm-bar suturing system (with video). Surg Endosc 2014; 28: 683-690

[7] Inoue H, Ikeda H, Hosoya T et al. Submucosal endoscopic tumor resection for subepithelial tumors in the esophagus and cardia. Endoscopy 2012; 44: $225-230$
[8] Xu MD, Cai MY, Zhou PH et al. Submucosal tunneling endoscopic resection: a new technique for treating upper gastrointestinal submucosal tumors originating from the muscularis propria layer. Gastrointest Endosc 2012; 75: 195-199

[9] Li QL, Chen WF, Zhang C et al. Clinical impact of submucosal tunneling endoscopic resection for the treatment of gastric submucosal tumors originating from the muscularis propria layer (with video). Surg Endosc 2015; 29: 3640 - 3646

[10] Yahagi N, Uraoka T, Ida Y et al. Endoscopic submucosal dissection using the flex and the dual knives. Techn Gastrointest Endosc 2011; 13: $74-78$

[11] Goto O, Sasaki M, Akimoto T et al. Endoscopic hand-suturing for defect closure after gastric endoscopic submucosal dissection: a pilot study in animals and in humans. Endoscopy 2017; 49: $792-797$

[12] Inoue H, Minami H, Kobayashi Y et al. Peroral endoscopic myotomy (POEM) for esophageal achalasia. Endoscopy 2010; 42: 265-271

[13] Oyama T. Counter traction makes endoscopic submucosal dissection easier. Clin Endosc 2012; 45: 375 - 378

[14] Sumiyama K, Gostout CJ, Rajan E et al. Submucosal endoscopy with mucosal flap safety valve. Gastrointest Endosc 2007; 65: 688-694

[15] Schmidt A, Beyna T, Schumacher B et al. Colonoscopic full-thickness resection using an over-the-scope device: a prospective multicentre study in various indications. Gut 2018; 67: $1280-1289$ 